\title{
Evolución paleoambiental del sector meridional del Parque Nacional de Doñana desde el Plioceno Inferior a la actualidad
}

\author{
Ostracods as palaeoenvironmental tracers: evolution of the southern \\ area of the Doñana National Park from the Lower Pliocene to Recent
}

\author{
F. Ruiz¹, M. Abad ${ }^{1}$, M. Pozo², M.I. Carretero ${ }^{3}$, J. Rodríguez Vidal' ${ }^{1}$, L.M. Cáceres ${ }^{1}$, E. \\ Font $^{4}$, M.L. González-Regalado ${ }^{1}$, A. Toscano ${ }^{1}$, E. X. García ${ }^{5}$
}

\section{RESUMEN}

\begin{abstract}
El estudio de los materiales geológicos y las asociaciones de ostrácodos presentes en un testigo largo obtenido en el sur del Parque Nacional de Doñana (SO de España) permite inferir una evolución paleoambiental desde medios marinos someros (Plioceno Inferior) hasta un lagoon salobre (Pleistoceno Superior-Holoceno) y la implantación de sistemas eólicos ( $<1.900$ años BP), con un estadio intermedio aluvial durante la mayor parte del Pleistoceno. En el Holoceno Superior, se detecta un evento tsunamigénico caracterizado por la erosión de sedimentos eólicos y su depósito sobre medios submareales.
\end{abstract}

Palabras clave: Ostrácodos, Paleoambientes, Plioceno-Actual, Sur Parque Nacional Doñana, España

\section{ABSTRACT}

The analysis of the geological units and the ostracod assemblages of a long core collected in the southern Doñana National Park (SW Spain) permits to deduce an evolution from shallow marine palaeoenvironments (Lower Pliocene) to a brackish lagoon (Upper Pleistocene-Holocene) and the deposit of aeolian sediments (<1900 yr BP), with an intermediate alluvial stage during the Pleistocene. In the Late Holocene, a tsunamigenic event was detected, with the erosion of aeolian sediments and a subsequent deposit on subtidal environments.

Key words: Ostracods, Palaeoenvironment, Pliocene-Recent, S Doñana National Park, Spain

\section{Introducción}

El registro geológico del Parque Nacional de Doñana ha sido objeto de numerosas investigaciones en las últimas dos décadas, que han puesto de manifiesto la complejidad de su evolución geomorfológica, la presencia de depósitos tsunamigénicos o la contaminación derivada de actividades mineras milenarias (Borja et al., 1999; Carretero et al., 2011). La mayoría de estas investigaciones se han centrado en los materiales holocenos de este registro, y especial- mente aquéllos depositados desde la Transgresión Flandriense, que tuvo lugar en esta área hace unos 6.500 años aproximadamente (Zazo et al., 1994).

Recientemente, Salvany et al. $(2010,2011)$ han diferenciado las distintas formaciones que componen el relleno del Parque desde el Plioceno Inferior, basándose en el estudio de numerosos testigos continuos. En este trabajo, se analizan los ostrácodos de uno de ellos, obtenido en la flecha de Doñana. A partir de su análisis, se pretende inferir la evolución paleoambiental de este sector en los últimos 5 millones de años.

\footnotetext{
${ }^{1}$ Dpto. Geodinámica y Paleontología, Universidad de Huelva. Avda. Tres de Marzo, s/n, 21071-Huelva. E-mail: ruizmu@uhu.es

2 Dpto. Geología y Geoquímica, Facultad de Ciencias, Universidad Autónoma de Madrid, 28049 Madrid.

${ }^{3}$ Dpto. Cristalografía, Mineralogía y Química Agrícola, Facultad de Química, Universidad de Sevilla, 41012 Sevilla.

4 IDL-Facultade de Ciencias da Universidade de Lisboa, Portugal.

${ }^{5}$ Dpto. Botánica y Zoología, Universidad de Guadalajara, 45510-Zapopán, México.
} 


\section{El Parque Nacional de Doñana}

El Parque Nacional de Doñana es una de más importantes Reservas de la Biosfera de Europa, con más de 50.000 ha que incluyen marismas fluviosalobres, levees fluviales, lagunas de aguas dulces (localmente llamadas "lucios") y una intrincada red de cauces fluviales y canales tributarios (fig. 1). La topografía de estas zonas internas es muy plana y apenas sobresale unos metros sobre el nivel del mar, coincidiendo con la presencia de crestas bioclásticas y arenosas, procedentes en algunos casos de la acción de tsunamis holocenos (p.e. Ruiz et al., 2005).

Estas zonas internas están protegidas por la flecha de Doñana, que se eleva por encima de los 15 $\mathrm{m}$ en algunos sectores. En su evolución, se han podido distinguir distintas fases de progradación durante el Holoceno Superior (fig. 1: $\mathrm{H}_{2}-\mathrm{H}_{4}$; Dabrio et al., 1999).

\section{Metodología}

El testigo estudiado fue obtenido en el año 2000 por el Instituto Geológico y Minero de España en el denominado "Corral de la Marta", situado en la flecha de Doñana (fig. 1: CM; 289,5 m; X: 732,572; Y: 4083,562; Z: $11 \mathrm{~m})$. En una primera etapa, se procedió a la diferenciación de los distintos materiales que lo componen. A continuación, se obtuvieron 35 muestras para el análisis de los ostrácodos y sus asociaciones. Un total de $50 \mathrm{~g}$ de cada una de ellas fueron levigados por un tamiz de $63 \mu \mathrm{m}$, con el estudio taxonómico de todas las valvas y caparazones presentes.

Finalmente, se seleccionaron dos muestras del tramo superior del testigo (Beta 228876: -29 m; Beta 228874: $-10,8 \mathrm{~m}$ ) para su datación mediante $14 \mathrm{C}$ en Beta Analytic Laboratory (Miami, USA). Estas muestras fueron calibradas con el programa CALIB 6.0. Los resultados corresponden a las edades calibradas con un intervalo de error de $2 \theta$, con la aplicación del efecto reservorio deducido por Soares \& Martins (2010) para este área. Las restantes edades incluidas en la figura 2 proceden de Salvany et al. (2011).

\section{Resultados y discusión}

Los 3 metros inferiores del testigo CM están constituidos por limos glauconíticos con algunos niveles lumaquélicos de ostreidos. Los ostrácodos

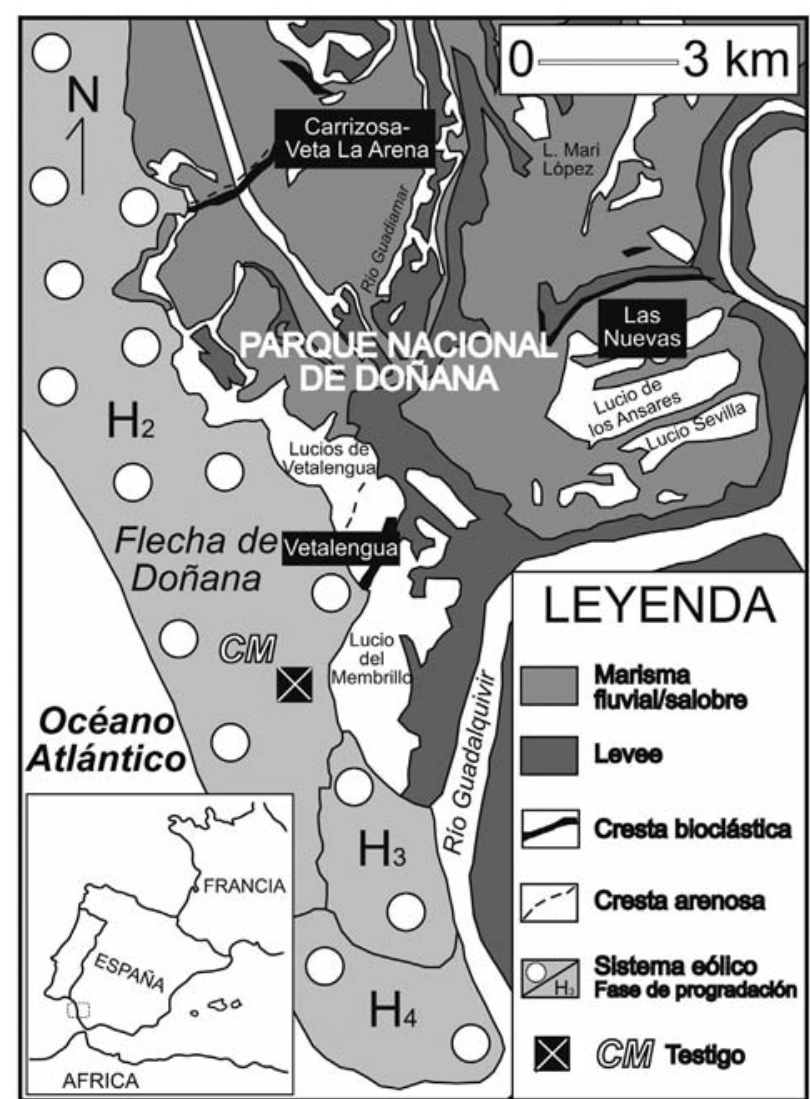

Fig. 1.-Geomorfología general del sector meridional del Parque Nacional de Doñana, con la localización del testigo CM. En negrita, nombre local de determinados sectores del Parque (p.e., Las Nuevas).

son muy escasos y están representados por Carinocythereis carinata y algunos raros ejemplares del género Loxoconcha. Estas características asimilan este tramo con los niveles basales glauconíticos de la Fm. "Arenas de Huelva" (Civis et al., 1987), que se habrían depositado en un medio marino infralitoral a circalitoral durante el Plioceno Inferior (Ruiz \& González-Regalado, 1996).

Esta correlación se ve reforzada por la presencia de niveles lumaquélicos fuertemente cementados de moluscos intercalados entre limos arenosos en los siguientes $65 \mathrm{~m}$, un rasgo típico de la parte superior de esta formación. Los ostrácodos presentes son propios de medios marinos someros (p.e. Hiltermannicythere retifastigata, Nonurocythereis seminulum; Ruiz et al., 2008) y la periódica acumulación de moluscos se debería a la acción de tormentas (González Delgado et al., 1995).

Los siguientes 80 m (-220 a -140 m) están formados por areniscas blancas a amarillentas (escala 


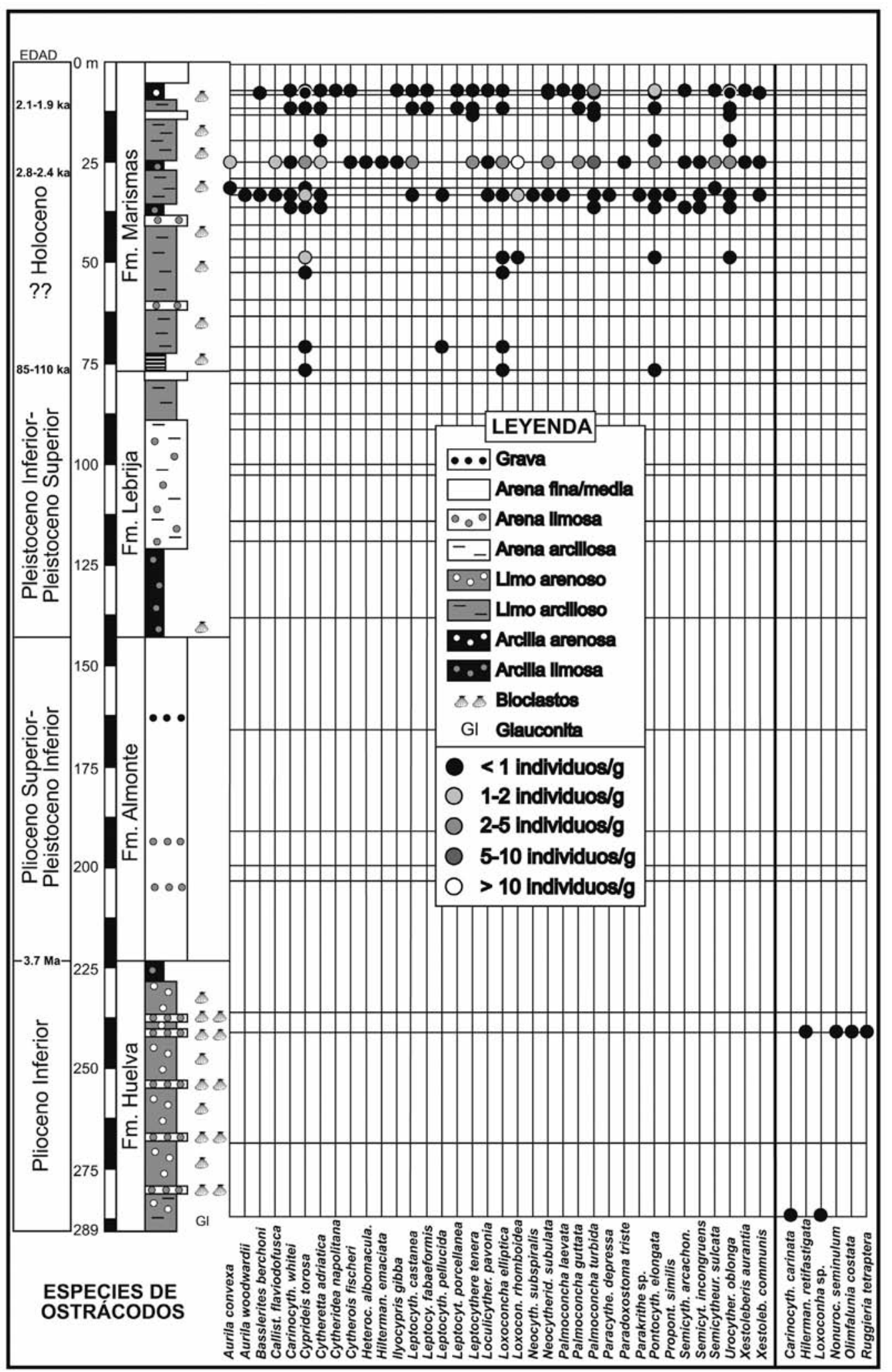

Fig. 2.-Sección sintética del testigo CM (modificada de Salvany et al. (2011) y Rodríguez Vidal et al. (2011), con inclusión de la abundancia relativa de las especies de ostrácodos en las muestras seleccionadas. Edades tomadas de Salvany et al. (2011), excepto las dos superiores (ver metodología). Las líneas horizontales coinciden con las muestras analizadas para el estudio de los ostrácodos. 
Munsell: $10 \mathrm{Y}$ 8/2), que pueden contener pequeños niveles anaranjados más arcillosos (10YR 6/6) en la parte inferior e incluyen finos espesores de lutitas negras con nódulos ferruginosos y restos vegetales carbonizados en su parte superior. Estos materiales han sido incluidos por Salvany et al. (2011) dentro de la Fm. "Almonte", con una edad comprendida entre el Plioceno Superior y Pleistoceno Inferior. Representarían la implantación de un sistema aluvial sobre un relieve previo neógeno. Estos depósitos carecen de ostrácodos (fig. 2).

El tramo suprayacente (-140 a -119 m) está dominado por lutitas de colores diversos $(5 \mathrm{Y} 7 / 2 ; 10 \mathrm{YR}$ 6/6), a veces con nódulos ferruginosos y restos vegetales carbonizados. A techo (-119 a $-89 \mathrm{~m})$, dominan las arenas amarillentas (10 YR 7/4; 5Y $8 / 4)$, que dan paso a lutitas y arenas amarillentas a techo $(-89$ a $-76 \mathrm{~m})$. Estos tres intervalos constituirían la Fm. "Lebrija" (Pleistoceno Inferior-Superior), con sedimentos aluviales distales (parte inferior) y proximales (parte superior) (Salvany et al., 2011). No presentan ostrácodos.

Los 76 m superiores del testigo CM (Fm. Marismas; Salvany et al., 2011) presentan una alternancia de limos arcillosos grisáceos (10YR 6/2) y, en menor medida, arcillas negras con abundante materia orgánica. Las asociaciones de ostrácodos de la parte basal $(-76$ a $-40 \mathrm{~cm})$ están dominadas por Cyprideis torosa y Loxoconcha elliptica, con Pontocythere elongata y Urocythereis oblonga como especies secundarias. Estas formas son típicas de lagunas costeras someras $(<10 \mathrm{~m}$ de profundidad) con conexión marina restringida, sometidas a importantes fluctuaciones de salinidad (Ruiz et al., 2006).

Hacia techo (-40 a $-6,5 \mathrm{~m})$, aumenta la densidad y diversidad de las asociaciones de ostrácodos, con proporciones similares de especies típicas de lagunas salobres (C. torosa, L. elliptica, Leptocythere spp.) y marinas someras (P. elongata, $P$. guttata, Semicytherura spp.) (Ruiz et al., 1997, 2006). Ello indicaría el tránsito hacia condiciones lagunares más abiertas, próximas a los canales de conexión con la plataforma adyacente (p.e., inlets) y una elevación relativa del nivel marino en esta zona. Ello podría coincidir con la subida progresiva del nivel marino a finales del Pleistoceno y el máximo de la trangresión flandriense en este sector (6.500 años; Zazo et al., 1994).

En este tramo, se detecta el depósito puntual de materiales eólicos, $(-17 \mathrm{a}-14 \mathrm{~m})$, formados por arenas amarillentas de grano fino a medio (color 10YR 8/2 a 10YR 6/2) sobre los sedimentos limo- sos submareales hacia los 2.200 años BP (fig. 2), Estas arenas presentan una ausencia casi total de valvas y caparazones de ostrácodos.

Esta pulsación procedería de la erosión de la flecha de Doñana producida por los tsunamis que acaecieron entre 218-210 años BC ó 2.200 años BP aproximadamente, de acuerdo con estudios geológicos pluridisciplinares (sedimentología, mineralogía, geoquímica, geomorfología) realizados en este área (Rodríguez Vidal et al., 2011). Una posible consecuencia es la presencia de una superficie erosiva que atraviesa la flecha en las proximidades del testigo CM (Rodríguez Ramírez et al., 1995), así como la presencia de una etapa erosiva entre dos fases de progradación, tanto en este spit como a nivel regional en este periodo (Zazo et al., 1994).

El depósito de estos materiales eólicos ocasionaría la colmatación parcial del canal de comunicación marina del lagoon, si bien este canal continua activo como se infiere de los materiales suprayacentes, de edades comprendidas entre 2.1 ka y 1.9 ka (fig. 2). Posteriormente, se produciría su emersión y la implantación progresiva de marismas (fig 2: arcillas superiores).

En los 6,5 m superiores ( $<1.900$ años BP), aparece un paquete de arenas amarillentas claras (10YR $8 / 2$ ), poco cementadas, moderadamente seleccionadas y muy ricas en cuarzo. No contienen ostrácodos ni fragmentos de macrofauna. Estas arenas tienen un origen eólico y constituyen la mayor parte de registro geológico de la flecha de Doñana. Se depositarían sobre los depósitos previos de marisma.

\section{Conclusiones}

El análisis de los materiales geológicos y las asociaciones de ostrácodos del testigo CM permite efectuar una aproximación a la reconstrucción paleoambiental del sector meridional del Parque Nacional de Doñana desde el Plioceno Inferior hasta la actualidad. Durante el Plioceno InferiorSuperior, se produce el tránsito desde medios marinos infralitorales, sometidos a periódicas tormentas, que caracterizan a la Fm. "Arenas de Huelva", a los medios aluviales que conforman los sedimentos suprayacentes. Dentro de estos últimos, existe una transición desde las zonas más distales (Fm. "Almonte") a las áreas más proximales (Fm. "Lebrija") durante el Pleistoceno. Estos medios aluviales se caracterizan por la ausencia de ostrácodos. 
El final del Pleistoceno y el Holoceno suponen la implantación inicial de una laguna salobre, inicialmente restringida y posteriormente con una conexión marina más amplia, con un depósito de sedimentos eólicos de origen tsunamigénico hacia los 2.200 años BP sobre medios submareales, que son parcialmente colmatados. Finalmente, se produce la emersión del área, el desarrollo de marismas y la implantación final de cordones eólicos con posterioridad a los 1.900 años $\mathrm{BP}$.

\section{AGRADECIMIENTOS}

Este trabajo ha sido financiado por los proyectos CGL201015810 y CGL2012-30875, un proyecto de la Fundacao para a Ciencia e a Tecnologia (PTDC/CTE-GIX/110205/2009), un proyecto de Excelencia de la Junta de Andalucía subvencionado con fondos FEDER (SEJ-4770) y tres grupos de investigación del Plan Andaluz de Investigación, Desarrollo e Innovación (RNM-238, RNM-293 y RNM-349). Es una contribución a los proyectos IGCP 526, 567 y 588. Los autores agradecen al Dr. Josep María Salvany y a dos revisores anónimos sus útiles consejos, que han contribuido a la mejora del manuscrito final, así como al Dr. José María Cebriá por sus aportaciones editoriales.

\section{Referencias}

Borja, F.; Zazo, C.; Dabrio, C.J.; Díaz del Olmo, F.; Goy, J.L. \& Lario, J. (1999). Holocene aeolian phases and human settlements along the Atlantic coast of southern Spain. The Holocene, 9: 333-339. doi:10.1191/095968399668924476

Carretero, M.I.; Ruiz, F.; Pozo, M.; Rodríguez Vidal, J.; Muñoz Pichardo, J.M.; Cáceres, L.M.; Gómez, F.; Campos, J.M.; González-Regalado, M.L. \& Olías, M. (2011). Trace Elements in Holocene Sediments of the Southern Doñana National Park (SW Spain): Historical Pollution and Applications. Environmental Earth Sciences, 64: 1215-1223. doi:10.1007/s12665-011-0936-9

Civis, J.; Sierro, F.J.; González Delgado, J.A.; Flores, J.A.; Porta, J. \& Valle, M. F. (1987). El Neógeno marino de la provincia de Huelva: Antecedentes y definición de las unidades litoestratigráficas. In: Paleontología del Neógeno de Huelva (W Cuenca del Guadalquivir) (Civis, J., ed.). Universidad de Salamanca, Salamanca, 9-23.

Dabrio, C.J.; Zazo, C.; Lario, J.; Goy, J.L.; Sierro, F.J.; Borja, F.; González, J.A. \& Flores, J.A. (1999). Sequence stratigraphy of Holocene incised-valley fills and coastal evolution in the Gulf of Cádiz (southern Spain). Geologie en Minjbouw, 77: 263-281.

González-Delgado, J.A.; Andrés, I. \& Sierro, F.J. (1995). Late neogene molluscan faunas from the Northeast Atlantic (Portugal, Spain, Morocco). Geobios, 28: 459471. doi:10.1016/S0016-6995(95)80021-2
Rodríguez Ramírez, A.; Siljeström, P.; Clemente, L.; Rodríguez Vidal, J. \& Moreno, A. (1995). Caracterización de las pautas geomorfológicas de la flecha litoral de Doñana. Revista de Teledetección, 5: 1-6.

Rodríguez Vidal, J.; Ruiz, F.; Cáceres, L.M.; Abad, M.; González-Regalado, M.L.; Pozo, M.; Carretero, M.I.; Monge, A.M. \& Gómez, F. (2011). Geomarkers of the 218-209 BC Atlantic tsunami in the Roman Lacus Ligustinus (SW Spain): A palaeogeographical approach. Quaternary International, 242: 201-212.

Ruiz, F. \& González-Regalado, M.L. (1996). Les ostracodes du Golfe Mio-Pliocene du sud-ouest de l'Espagne. Révue de Micropaléontologie, 39: 137-151. doi:10.1016/S0035-1598(96)90038-0

Ruiz, F.; González-Regalado, M.L. \& Muñoz, J.M. (1997). Multivariate analysis applied to total and living fauna: seasonal ecology of recent benthic Ostracoda off the North Cádiz Gulf coast (southwestern Spain). Marine Micropaleontology, 31: 183-203. doi:10.1016/S0377-8398(96)00060-6

Ruiz, F.; Rodríguez-Ramírez, A.; Cáceres, L.M.; Rodríguez Vidal, J.; Carretero, M.I.; Abad, M.; Olías, M. \& Pozo, M. (2005). Evidence of high-energy events in the geological record: Mid-Holocene evolution of the southwestern Doñana National Park (SW Spain). Palaeogeography, Palaeoclimatology, Palaeoecology, 229: 212-229. doi:10.1016/j.palaeo.2005.06.023

Ruiz, F.; Abad, M.; Galán, E.; González, I.; Aguilá, I.; Olías, M.; Gómez Ariza, J. L. \& Cantano, M. (2006). The present environmental scenario of El Melah Lagoon (NE Tunisia) and its evolution to a future sabkha. Journal of African Earth Sciences, 44: 289-302. doi:10.1016/j.jafrearsci.2005.11.023

Ruiz, F.; Abad, M.; Civis, J.; González Delgado, J.A.; González-Regalado, M.L. \& García, E.X.M. (2008). Pliocene ostracods of southwestern Spain. Geobios, 41: 845-859. doi:10.1016/j.geobios.2007.07.004

Salvany, J.M.; Mediavilla, C. \& Rebollo, A. (2010). Las formaciones Plio-Cuaternarias de El Abalario, en el litoral de la provincia de Huelva (España). Estudios Geológicos, 66:209-225. doi:10.3989/egeol.39953.072

Salvany, J.M.; Larrasoaña, J.C.; Mediavilla, C. \& Rebo1lo, A. (2011). Chronology and tectono-sedimentary evolution of the Upper Pliocene to Quaternary deposits of the lower Guadalquivir foreland basin, SW Spain. Sedimentary Geology, 241: 22-39. doi:10.1016/j.sedgeo.2011.09.009

Soares, A.M.M. \& Martins, J.M.M. (2010). Radiocarbon dating of marine samples from Gulf of Cadiz: the reservoir effect. Quaternary International, 221: 9-12. doi:10.1016/j.quaint.2009.10.012

Zazo, C.; Goy, J.L.; Somoza, L.; Dabrio, C.J.; Belloumini, G.; Improta, S.; Lario, J.; Bardají, T. \& Silva, P.G. (1994). Holocene sequence of sea-level fluctuations in relation to climatic trends in the Atlantic-Mediterranean linkage coast. Journal of Coastal Research, 10: 933-945.

Recibido el 12 de junio de 2012 Aceptado el 15 de noviembre de 2012 Publicado online el 16 de abril de 2013 